\title{
A LEAN E-GOVERNANCE APPROACH TO MITIGATE CORRUPTION WITHIN OFFICIAL PROCESSES IN THE CONSTRUCTION INDUSTRY
}

\author{
Alaa Daramsis ${ }^{1}$, Karim Faour ${ }^{2}$, Lynn Richa Abdel Ahad ${ }^{3}$, \\ Ghadeer Salami ${ }^{4}$, and Farook Hamzeh ${ }^{5}$
}

\begin{abstract}
Delays in construction projects lead to litigation-related issues and waste of money, in addition to rescheduling task and deliveries to fit the newly delayed plan, which is no easy task to accomplish given the hundreds of activities on a typical construction site. One of the factors that subject the schedule to delays is the official processes performed at the public sectors. Lebanon is a country that has been plagued by corruption; official processes run at suboptimal levels and delay the day-to-day activities of citizens and projects across all industries. The concept of applying lean methods to mitigate this corruption is promising; as this is the first study in Lebanon to address this issue by employing a lean perspective. The aim of this paper is to analyze, assess, and formulate frameworks of official processes based on interviews conducted with professionals in the field who have experienced unexplained delays in their construction documents. In addition, this study develops a tailored e-governance strategy that would effectively lead to a lean revamp in the public sector in terms of service quality, transparency, and reliability. Moreover, it serves as the theoretical foundation for the transformative shift in the official processes in the Lebanon.
\end{abstract}

\section{KEYWORDS}

E-Governance; Lean IT; Work flow; Standardization, Work Structuring.

1 Undergraduate Student, Civil and Environmental Engineering Department, American University of Beirut, Lebanon, Phone +961 71637 393, akd12@ mail.aub.edu

2 Undergraduate Student, Civil and Environmental Engineering Department, American University of Beirut, Lebanon, Phone +961 71112 886, knf03@ mail.aub.edu

3 Undergraduate Student, Civil and Environmental Engineering Department, American University of Beirut, Lebanon, Phone +961 3538 176, gjs08@mail.aub.edu

4 Undergraduate Student, Civil and Environmental Engineering Department, American University of Beirut, Lebanon, Phone +961 70425 750, lar09@mail.aub.edu

5 Assistant Professor, Civil and Environmental Engineering Department, American University of Beirut, Beirut Riad El-Solh 1107 2020, Lebanon, Phone +961 1350000 Ext: 3616, Farook.Hamzeh@mail.aub.edu 


\section{INTRODUCTION}

Lean is minimizing waste and maximizing value to ultimately reach a perfect valuecreating process containing zero waste (LEI, 2017). Lean cities are those who apply lean principles to maximize citizens' value-added services and eliminate waste from their processes (Quintana, 2017). "Lean Governments are part of a growing trend of management tools designed to lower costs and improve the efficiency of government operations" (Scorsone, 2010). Lebanon is a country with high corruption rates that are magnified by the inefficient systems that are present, especially since corruptive actions have become embedded within the mentality of people (Awada, 2014).

This paper discusses corruption in Lebanon, and how the implementation of lean methodologies into its governmental sectors can prevent corruptive actions, achieve efficiency, and consequently save time and decrease unnecessary costs on construction projects.

The concept of lean cities is already being implemented in some countries. In Minnesota, for example, they have offices that are dedicated to continuous improvement that develop new ways for inspection that save on money and introduce new tools into their departments (Minnesota Government, 2017). Colorado is another state where the concept of lean governments was successfully implemented. The state's approach is made up of three pillars: cross-functional process improvements, localized process improvements, and everyday ideas and respect for people. This in turn led to remove 23 on-value adding steps and issuing permits quicker with fewer errors. Moreover, the transition that the Colorado Department of Transportation did to become paperless saved it $\$ 18,000$ a year as a consequence of digitizing the government and integrating lean applications to its IT (Codot, 2017). Another example would be the Connecticut Department of labor where they saved 119 steps, eliminated 1181 cycle time hours and saved $\$ 500,000$ in staff time over a year (Johnston, 2013). All the aforementioned figures were to highlight the positive correlation between applying lean practices and reducing waste.

Lebanon was the $136^{\text {th }}$ least corrupt nation out of 175 countries, costing the state an estimated $\$ 800$ million a year. Moreover, it scored 28/100 in a corruption indicator that ranges between zero (highly corrupt) and 100 (very clean), which is worse than the overall Middle East and North Africa's average score of 38/100 (Transparency International Corruption Perceptions Index,2016). This highly corrupt score was associated with the absence of presidential elections where corruption has extended to almost all governmental institutions with rare supervision (Lebanese Transparency Association, 2016).

A messy queue of people holding thick folders of official documents behind a counter is a familiar scene for anyone who goes to ministries or state intuitions in Lebanon. In light of the Lebanese bureaucracy, the experience of requesting any kind of official document could be confusing and annoying as people struggle to access basic governmental services. "Our system feels like it's still the same since the time of the Ottomans," Bassem Deaibess, a 35-year-old musician, told The Daily Star. "I lost my 
A Lean E-Governance Approach to Mitigate Corruption within Official Processes in the Construction Industry

passport in 2011 and the entire process, to get a new one, took over a month."(Nashed,2016).

Bribery is a critical aspect of corruption that exhausts not only the government treasury but also the citizens' money. Corrupt officials plunder the government treasury through processes' fees of legal and illegal transactions in the governmental departments (Beirutie, 2015). The reasons behind citizens' bribes reveal that 49 percent of Lebanese people offer bribes to speed up transactions, 19 percent pay bribes as gifts, 16 percent pay for cheaper services, and more than 16 percent perceive it as the only way to get the service done. When it comes to reporting corruption cases, more than half of Lebanese would prefer to stay silent rather than report a corruption incident in fear of becoming victims for reporting. Also, they believe that reporting such incidents will not make perpetrators get punished (Awada,2014). In addition, "the Lebanese developed tools such as Wasta as methods that can assure trust in their daily transactions (Harfouche\& Robbin, 2012). "Wasta" comes from an arabic root conveying the idea of "middle" and a wasta is someone who acts as a go-between and use intermediaries. The intermediaries in case of wasta must be someone with influence but not necessarily a relative or even a close friend (Whitaker, 2009).

In the light of the problems mentioned above, e-governance via digitizing governmental system provides a tool that enables the control over the process and allows the integration of lean principles. This tool is a first step of applying a long term strategy that adds value to the customer and removes wastes, thus providing the philosophy foundation of the 4P model (Liker, 2013). E-governance also allows more transparency in the system, limiting both "Wasta" and bribery and improving the second step in the 4-P model called process (Alawadhi\& Morris, 2009). Moreover, this clear and transparent process will raise problems to the surface and will challenge employees to think outside the box and provide creative solutions (Liker, 2013). This is the third part of the 4-P model that includes growing leaders that live the philosophy. The last step is Kaizen, meaning continuous improvement, and is ensured by an E-government since it would be the first major change implemented to move to a better system.

The increasing rate of corruption in the Lebanese government makes it more urgent to introduce lean techniques into the public sector. Just as it has been implemented in other cities for more than 15 years, the time has come to start implementing lean methodologies in Lebanon. Therefore, this paper introduces a lean IT strategy that aims at creating a flowing balanced process that provides transparency and supervision to prevent corrupt intentions by employees.

\section{METHODOLOGY}

This paper summarizes exploratory research about corruption within official processes in Lebanon and the impact of applying lean tools on reducing the incurred document delays. Research involves both a survey of available literature about existing lean cities and short interviews to determine the current process steps and the impact of document delays on the construction projects. Interviews were conducted with construction engineers and a document tracker that has been working in the field for more than 30 years. The 
document tracker's experience reflects the situation of Lebanese official centers in general since he has worked in several districts across Lebanon.Interviewed personnel were asked about the delays they encountered, the reasons behind these delays and possible solutions to prevent them. The problem with the current governmental practices was found to be its high dependency on human decisions and acts as well as its lack of supervision.

The strategy proposed involved an arbitrary document of "Type X" from start up until completion. The research process comprised the following steps: evaluating current practices in completing documents as well as developing a lean IT strategy that aims at improving the latter practices. The strategy methods include the achievement of a more efficient process with transparency that allows the citizen to view the status of his documents. Moreover, the document has both a time tracking system to determine if it is being delayed and an online payment mode to prevent any form of bribery.

This research reflects guidelines for improvements in Lebanese official document processes through adopting a lean IT approach and accordingly do not pertain to any practical testing or application of these guidelines.

\section{CURRENT STATE}

The typical procedure for issuing a construction permit includes 3 major entities. It is first submitted to the order of engineers where it takes about two to three days to be reviewed and signed by a mechanical, electrical and executive engineer. After their approval it would be taken to the urban planning office where it is signed by the 3 engineers as well, each has to go over the drawings. This process usually takes 10 days. Finally it is taken to the municipality of that area, where they conduct weekly meetings to review submitted requests of that week. However, if they find any problem it is put on hold for further check to be discussed again in next week's meeting.

The average time for issuing the permit is found to be two months, since other that these mentioned durations the document might be delayed for corruptive reasons waiting for "gifts" to speed up the process. After the construction phase, residential permits must be issued to register apartments for the owners. The official time for this permit is stated as ten days, where it passes through five stages to check that the constructed facility coincides with the designs and that prices are adequate.

One can clearly notice from the steps listed above that the process is vulnerable to manipulation by the employees performing it; especially when supervision is absent. And a great proof of that is the fact that it can be performed within one day if a bribe is offered.

F.H. is a document tracker who has been in field for over 30 years. He believes that bribery has become the guaranteed path to perform any service in a much shorter time, and that employees in public sectors manipulate their positions for personal interests. $\mathrm{He}$ stated that sometimes they might fabricate illegal intentions or even claim that a document is missing just to provoke the owner to offer a bribe for him. The following table presents the results of short interviews conducted with engineers with their experience with construction project delays due to corruption in official processes. 
A Lean E-Governance Approach to Mitigate Corruption within Official Processes in the Construction Industry

Table 1 Survey of Governmental Inefficiency Effects

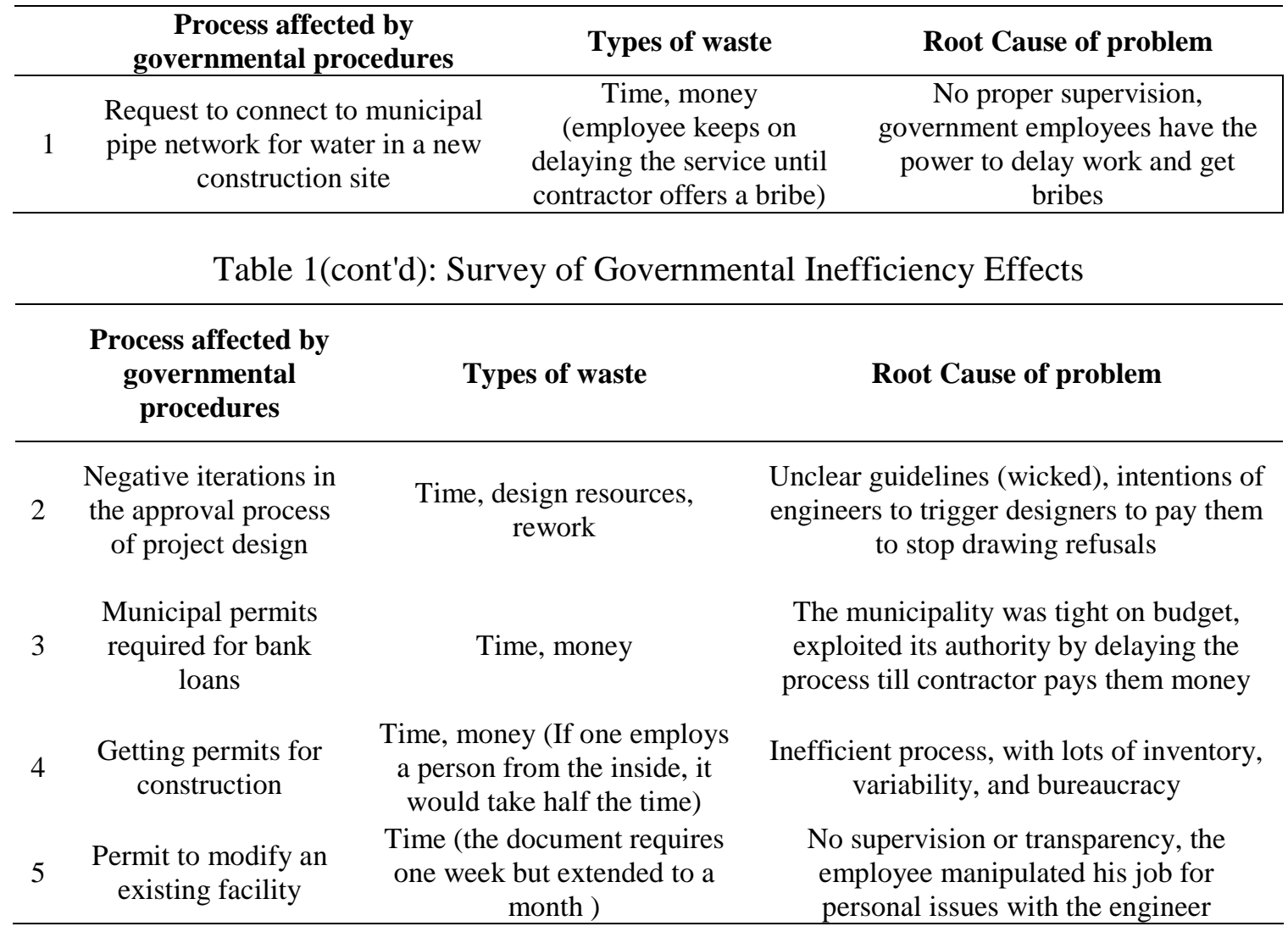

The first step in solving the problems stated above is to establish clear simple guidelines that cannot be manipulated. Second, processes must be digitized to enable the control over them and the introduction of lean principles to make them more efficient. Therefore, a strategy must be followed to mitigate corruptive actions, make the process more efficient, and subject it to further improvements.

\section{DEVELOPING A STRATEGY TO MITIGATE CORRUPTIVE ACTIONS}

In order to develop an effective strategy to mitigate the effect of corruption within official processes, two strategies must be followed to illustrate the methods in which an impactful change can be achieved.

\section{BEHAVIORAL STRATEGY}

The first is a behavioral strategy that aims to positively shift the mindset of public employees, making them potential agents of change in terms of the quality and reliability of the public services they perform. Before attempting to change anything, one must acknowledge the fact that change does not come easily; people are, by nature, afraid of change, and become unconsciously resistant to any idea that is forced on them without their personal approval and against their personal interests. This strategy is not the 
contribution of this paper; however, it is important to state it since it covers one of the 4P's of the Toyota Way model, which is investing in people in such a way that the organizational philosophy becomes incorporated within their contributions at work.

\section{LEAN IT STRATEGY}

Moving on from the lean behavioral strategy, we reach the second part of the "lean revamp" that aims to mitigate the effects of corruption in official processes. A lean IT strategy is proposed to block the means for bribing and the unlawful delay of a document request. It provides transparency that enables the citizen to monitor the progress of his/her document, track delay, and report it. It also aims at monitoring the progress of each employee and helps apply the rewards/penalty system.

\section{FOUNDATIONS OF LEAN IT}

It is important that one develops a proper infrastructure that will be the foundation for the Lean IT strategy that will be discussed later on. This foundation is in the form of an Enterprise Resource Planning (ERP) System that breaks down barriers in terms of information silos and enables the swift and real-time transfer of information between different governmental entities involved. Lebanese ministries always have interdisciplinary work among them that requires them to connect with each other in an efficient manner, so an ERP system would be essential to maintain a proper flow of information among them.

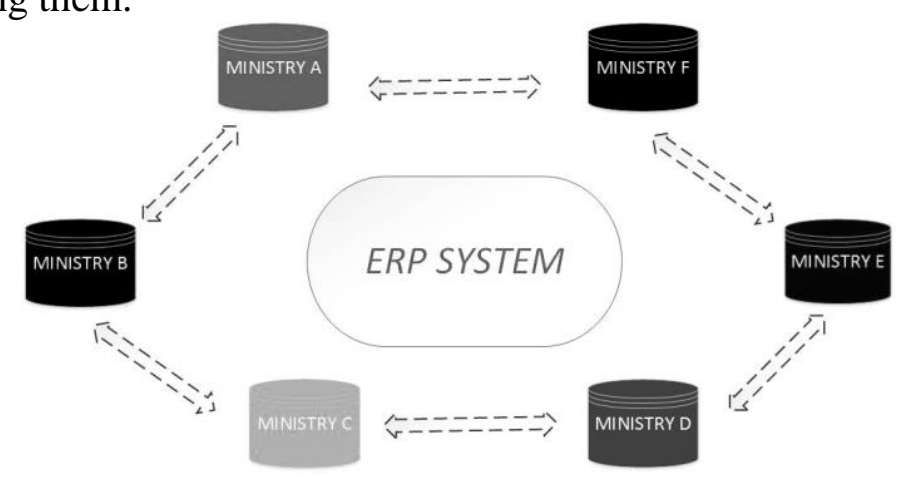

Figure 1: Enterprise Resource Planning (ERP) System

To properly illustrate this section, we will take a case study pertaining to how corruption within official processes affects the construction industry and create a Lean IT strategy that, once implemented, will tend to the issue of mitigating corruption. This case study involves tracking the passage of an arbitrary document "Type X" in the following proposed Lean IT system as it moves from station to station to be completed and handed over to the customer.

\section{PHASES OF LEAN IT}

The lean IT strategy is comprised of two phases, the first ensures that all prerequisites for submitting a certain document are ready and the second phase ensures that no document 
A Lean E-Governance Approach

to Mitigate Corruption within Official Processes in the Construction Industry

is delayed beyond a specified allowable time frame. Both phases will be thoroughly discussed in the following sections.

\section{Phase I:}

As shown in figure 2, a new, arbitrary construction document request of "Type $X$ " is generated online and the system gives the user an order number. This request will enter the proposed system and will pass through two checkpoints. The first checkpoint is the "Document Checker" that makes sure that he has all prerequisites ready, i.e., all associated documents that are required to fully process his request. Such items include, for example, his ID as well as project information about the project (municipality permit, ownership contract, electricity and water permits...), size of the project, and so on. If any document is missing, a notification will be sent to the user telling him to submit the missing document(s) for his request to be considered, or else it would be discarded. Once all documents are ready, the request passes by the second checkpoint: the online payment.

Online payment is of utmost importance because it will block the means to get a physical bribe. The system will detect only a certain amount of credit that has to be satisfied. If the customer pays less, a notification will be sent to him to pay the remaining amount, and if he pays more, the system will not accept it. After payment is complete, the document enters the second phase of the system, Phase II.

\section{Phase II:}

The document will now enter into a one-piece flow, First-In-First-Out (FIFO) Queueing System. Considering the example of the arbitrary construction document "Type $X$ " above, assume that this type of document usually takes around seven days to complete and passes by two checkpoints, annotated in figure 3 as Checkpoint A and Checkpoint B. (These checkpoints represent the different entities that the document passes through and might not necessarily be in the same place; checkpoint A may be the Ministry of Labor and checkpoint B may be the Ministry of Transportation, for instance). These seven days are split as follows: Part of the work requires three days at Checkpoint A and four days at Checkpoint B. Document requests are sent to Checkpoint $\mathrm{A}$ in the aforementioned manner. One can take the scenario that there is only one workstation available to process documents. These documents will be tracked according to the number of days they spend at a workstation; three designated zones are present to account for the time spent in processing: The green color (designated as $\mathrm{G}$ ) tells us that the time spent in a workstation is acceptable, the yellow color (designated as $\mathrm{Y}$ ) tells us that the time spent at a workstation is fair, and the red color (designated by R) indicates that the document has spent more than it should have at the workstation. Now, assume that document requests are spending more than they should have at workstation 1 of Checkpoint A. This tells management that there is a bottleneck at that point of the process, hence proper action must be taken to remove this waste, especially since we will have an increase in amount of work waiting on workers, which according to lean, is waste. However, given governmental budget constraints, one can opt to either provide extra training to the worker(s) involved at the station or can add to the number of workstations available to increase the processing rate of document requests and overall efficiency of the checkpoint 
itself. The same logic applies for any other existing checkpoints involved in process, regardless if these checkpoints belong to the same ministry or not. The system records the productivity of workers to assess their contribution to the checkpoint in order to have a continuous assessment of the system's performance and the possibility of improvement.

One can draw out an analogy between how tellers at a bank operate and how documents are processed in the proposed system. A bank teller is expected to complete, for example, no less than 30 requests per day assuming there is an infinite amount of customers available in the queue to be serviced. When management records the amount of requests completed versus what was expected of them to complete, they have to identify the source and nature of the error and correct it accordingly in the case of suboptimal performance, and reward those who have proven themselves competent over time.

From the customer's end, they can check the status of their documents online to see whether they have been processed or not. They simply enter their document order number given to them at the start of the process and an output screen will appear showing them a real-time tracking of their application and how many tentative days remain until their document is ready for collection. This estimated time of completion will change based on the overall productivity of checkpoints which are detected automatically by the system.

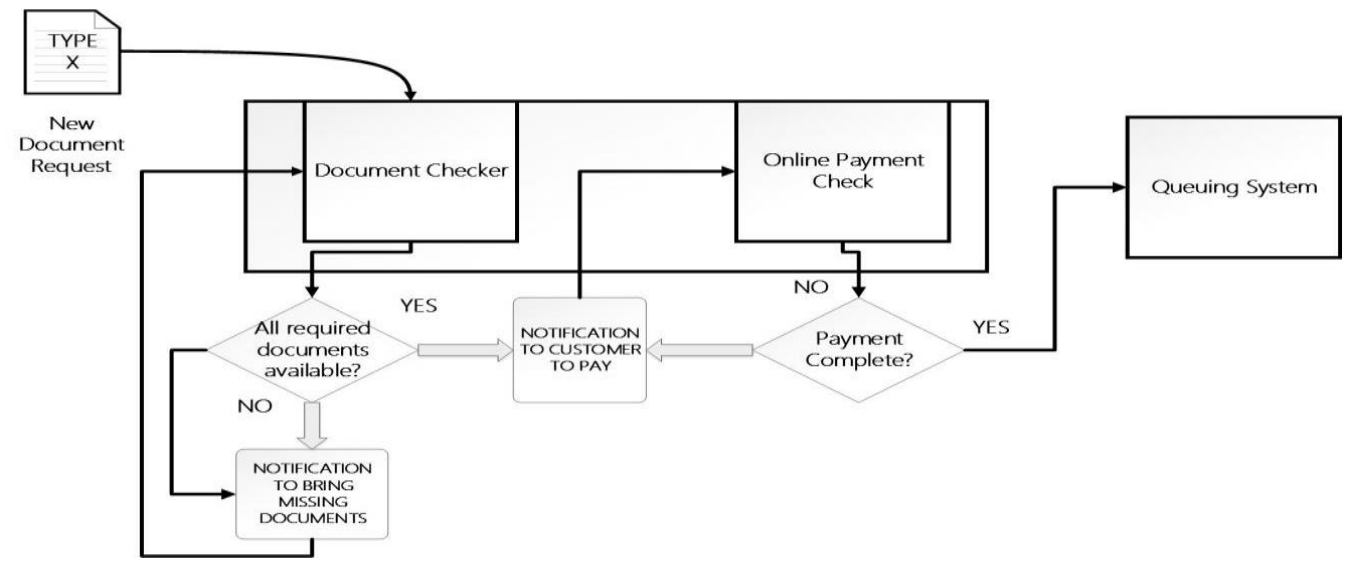

Figure 2: Phase I of the Lean IT Strategy
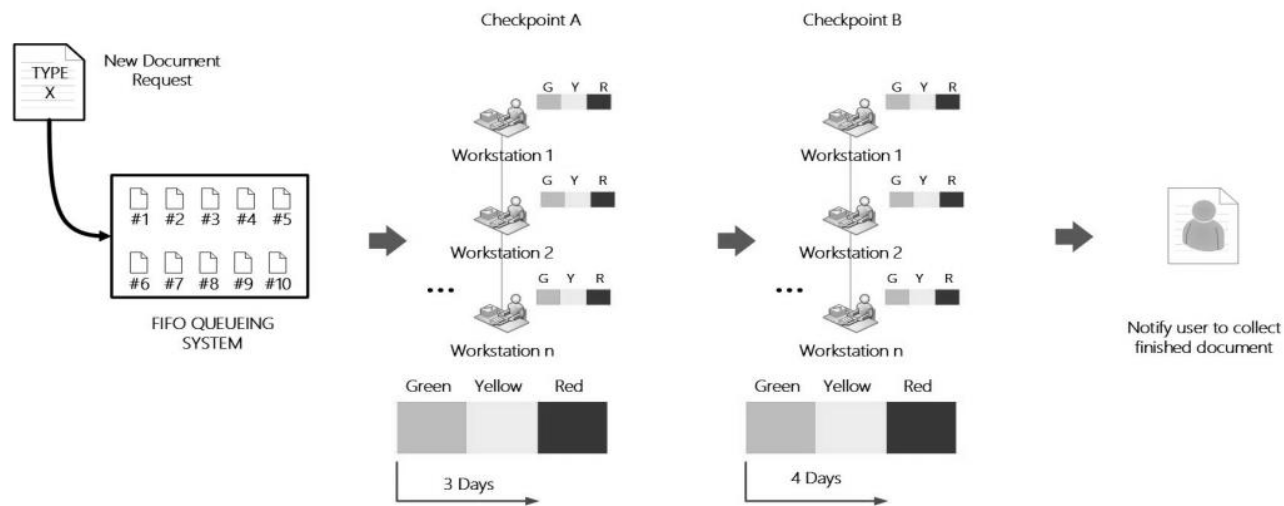

Figure 3: Phase II of the Lean IT Strategy 
A Lean E-Governance Approach

to Mitigate Corruption within Official Processes in the Construction Industry

\section{DISCUSSION}

Employees in the public sector come from a variety of backgrounds, cultures, norms, mindsets, and levels of qualification. Some believe that since they are Lebanese official employees, then they should perform at the same level the government does, which in this case, is very much underperforming and corrupt. This, in turn, will encourage them to negatively exercise the power their positions give them by demanding or accepting bribes or gifts in exchange for a quicker service. Furthermore, the role of "strong connections" to authoritative people and intimidating leaders has its role to play in terms of who gets which position and with what salary. It cannot be assumed that this will completely end just by applying an IT strategy because practically speaking, it will not. Hence, it is also very important to take into consideration the design for a lean behavioral strategy that will institutionalize a cultural change. This would be like watering the trees of a garden. A garden would not be fruitful just by placing trees into its pots; however, it requires watering and proper care to have it sustained. Employees need methods for a behavioral strategy to be able to grow. Proper care and follow up are essential as well to prevent any disease from plaguing the present environment.

Therefore, a global approach must be taken to enable the creation of a behavioral strategy that aims to positively shift the mindset of public employees, making them potential agents of change in terms of the quality and reliability of the government services they perform.

Moreover, let us look at this behavioral strategy from another perspective using the 10-10-10 Rule (Welch, 2009). The 10-10-10 Rule will tell us to think of this strategy as follows: What effect will this behavioral strategy generate in 10 minutes, in 10 months, and in 10 years? In 10 minutes, it is obvious that if you approached a public employee with this strategy, he/she would tell you that you can try to implement it but no one will take it seriously. Now, imagine if this idea was being applied for 10 months. 10 months is not a sufficient time for implementing such a strategy, but one thing would be certain which is that at least part of the lean principles would be absorbed by employees, and an incremental change will definitely be visible in terms of overall performance. In 10 years of incorporating this value-adding, lean behavioral strategy, and taking into consideration the number of new employees that would have entered into this partially-shifted, new positive-mindset environment; a definite change would be noticeable because this culture will be integrated and handed down from one generation of employees to the next as it is being implemented. This paper would open up the possibility for more detailed research to implement effectively a strategy that targets the behavior of Lebanese governmental employees.

\section{CONCLUSIONS AND RECOMMENDATIONS}

In conclusion, it is fair to say that change will not come easily when it comes to changing the culture and mentality of Lebanese government employees, but it is only in adopting and staying committed to long-term lean strategies that will give us the opportunity to mitigate the amount of corruption in official processes. These strategies will start by removing inefficiencies of current procedures to end with improved processes that would 
keep improving with time. By that, we would elevate the standards and transparency within the Lebanese Construction Industry in a way that would motivate other industries to adopt such a lean mentality, and in return, advance at both a personal level as well as a country-wide level through promoting a sense of reliability and authenticity of work.

\section{ACKNOWLEDGMENTS}

We would like to thank the Civil and Environmental Engineering Department at the American University of Beirut, as well as all the individuals who gave us an insight from their own experiences regarding this paper's topic.

\section{REFERENCES}

Alawadhi, S., \& Morris, A. (2009). Factors Influencing the Adoption of E-government Services. Journal of Software, 4(6), 584-590.

Awada, A. (2014). Listen, Rida: Lebanon is drowning in corruption. Retrieved November 30,2017, from Al Mada Organization website: https://goo.gl/sctaCN.

Beirutie, N. (2015). Corruption affects public administrations in Lebanon. E-Government is the solution. from Al Deyar e-newspaper website: https://goo.gl/BGSQUq.

Choueiri, E., Choueiri, G., \& Choueiri, B. (2013). An Overview of e-Government Strategy in Lebanon. from November 30,2017, from International Arab Journal of e-Technology website: https://goo.gl/VPGPLw.

Codot.(2017).colorado. from business center:https://www.codot.gov/business/processimprovement

Harfouche, A., \& Robbin, A. (2012). Inhibitors \&enablers of public e-services in Lebanon. Journal of Organizational and End User Computing (JOEUC), 24(3), 45-68.

Johnston, Z. R. (2013). Lean in UK Government: internal efficiency or. Production Planning \& Control.

Lean Enterprise Institute.(2017).What is Lean. Retrieved November 29,2017, from Lean Enterprise Institute: https://www.lean.org/WhatsLean/

Liker, Jeffrey K. Toyota Way: 14 Management Principles from the World's Greatest Manufacturer. McGraw-Hill, New York, 2013.

Minnesota government (2017). Minnesota. from November 29,2017, from Department of Administration: https://mn.gov/admin/continuous-improvement/results/

Nashed, M. (2016). Lebanon waits for e-government. The Daily Star

Quintana, F. A. (2017).Ie. Lean Cities: from https://www.ie.edu/corporaterelations/insights/lean-cities/

Scorsone, E. A. (2010). New Development: What are the Challenges in Transferring Lean Thinking to Government? Public Money \& Management.

Stifi, F. G. (2017). Lean Anti-Corruption Toolkit. Greece: International Group for Lean Construction (IGLC).

"Transparency International Corruption Perceptions Index 2016" (2016). From Transparency International Website: https://goo.gl/HYYNRg.

Welch, S. (2009). 1010 10: A Life-Transforming Idea.

Whitaker, Brian. What's Really Wrong with the Middle East. Saqi, London, 2009. 